Published in final edited form as:

Wiley Interdiscip Rev Nanomed Nanobiotechnol. 2009 ; 1(5): 568-581. doi:10.1002/wnan.50.

\title{
Nanoporous membranes for medical and biological applications
}

\author{
Shashishekar P Adiga \\ spadiga@anl.gov Materials Science Division, Argonne National Laboratory, Argonne, IL 60439 \\ Chunmin Jin \\ Department of Biomedical Engineering, University of North Carolina at Chapel Hill, Chapel Hill, \\ NC 27599, USA \\ Larry A Curtiss \\ Materials Science Division and Center for Nanoscale Materials, Argonne National Laboratory, \\ Argonne, IL 60439, USA
}

Nancy A. Monteiro-Riviere

Center for Chemical Toxicology Research and Pharmacokinetics, North Carolina State University, Raleigh, NC 27606, and the Joint Department of Biomedical Engineering, University of North Carolina and North Carolina State University, Chapel Hill, NC 27599,USA

\section{Roger J Narayan}

Department of Biomedical Engineering, University of North Carolina at Chapel Hill, Chapel Hill, NC 27599, USA

\section{Abstract}

Synthetic nanoporous materials have numerous potential biological and medical applications that involve sorting, sensing, isolating and releasing biological molecules. Nanoporous systems engineered to mimic natural filtration systems are actively being developed for use in smart implantable drug delivery systems, bioartificial organs, and other novel nano-enabled medical devices. Recent advances in nanoscience have made it possible to precisely control the morphology as well as physical and chemical properties of the pores in nanoporous materials that make them increasingly attractive for regulating and sensing transport at the molecular level. In this work, an overview of nanoporous membranes for biomedical applications is given. Various in vivo and in vitro membrane applications, including biosensing, biosorting, immunoisolation and drug delivery, are presented. Different types of nanoporous materials and their fabrication techniques are discussed with an emphasis on membranes with ordered pores. Desirable properties of membranes used in implantable devices, including biocompatibility and antibiofouling behavior, are discussed. The use of surface modification techniques to improve the function of nanoporous membranes is reviewed. Despite the extensive research carried out in fabrication, characterization, and modeling of nanoporous materials, there are still several challenges that must be overcome in order to create synthetic nanoporous systems that behave similarly to their biological counterparts.

\section{Keywords}

Biosensing; Drug delivery; Implantable materials; Nanopores; Nano-scale membranes

Biomedical engineers have recently recognized that medical implants require well-defined and controlled interfaces. One of the major obstacles preventing the clinical application of active devices that perform biologically useful functions has been the reduction in function after implantation due to poor understanding of the implant-tissue interface [1,2]. It is still not possible to maintain long-term in vivo functionality of an active medical implant, even 
when the device demonstrates satisfactory in vitro performance for prolonged periods of time. The external surface of an active implant has to possess properties that are not comparable with those necessary for hip prostheses and other traditional structural implants.

The biomaterials community over the past fifty years has excelled at creating materials that are either completely inert (e.g., hip prosthesis) or completely biodegradable (e.g. resorbable sutures). The new challenge for biomaterials engineering is the development of materials that minimize cell adhesion, protein deposits, and encapsulation, since these biological reactions reduce the ability of active medical devices to interact with the biological environment. Biosensors and drug delivery implants are active medical devices that must be capable of function during use over the months, years, and possibly decades. These devices must exhibit functional stability under a wide range of biological conditions. The functional lifetime of an active medical device will be dramatically increased if biofouling (especially protein absorption) and inflammation are minimized.

In an effort to meet long-term physical and chemical stability requirements, researchers have looked for an ideal interface that allows selective molecular exchange between the host tissue and the implant, while protecting the latter against immune rejection. There are innumerable examples of nanoporous interfaces performing multiple functions in biological systems, for example, cell membranes separate the interior of the cell from its environment and tightly regulate molecular traffic in and out of the cells through nanometer scale protein pores. To give a specific example, a synthetic membrane around an artificial pancreas would allow the passage of glucose, oxygen and other small molecules, but exclude the passage of proteins and other large molecules. Perhaps the likelihood of success in designing biocompatible membranes with desired functionality will increase if an effort was made to mimic the function of biological membranes as closely as possible. As we move into the era of nanomedicine, the development of such smart nanoporous membranes becomes critical for a variety of implantable medical devices, including controlled as well as signalresponsive drug delivery [3], immunoisolation devices [4], and microdialysis systems [5].

In addition to potential applications in implantable devices, nanoporous materials because of their ability to discriminate molecules based on size, shape and interaction, have gained considerable prominence in the biomedical area in the development of miniaturized devices for biomolecular analysis [6]. Currently, biomolecules are routinely separated for bioanalytical purposes using porous gel structures either by gel filtration or by gel electrophoresis. In response to the push towards more efficient separation techniques functionalized nanoporous structures with ordered pores are extensively explored to accomplish sensing, sorting, releasing, and isolating biomolecules in implantable devices in addition to bioanalytical techniques.

The aim of this review is to present an overview of nanoporous materials highlighting both diagnostic and therapeutic biomedical applications as well as the key challenges to be addressed in realizing ideal synthetic membranes discussed above. Porous materials have long been used in many important industrial applications such as catalysis, membrane separation, and storage. In the literature, porous materials are often referred to as nanoporous if the pore size is between $1-100 \mathrm{~nm}$ [7]. While multiple terminologies have been used to describe these materials, for the sake of consistency we will use the term nanoporous to refer to all porous materials with pore size less than $100 \mathrm{~nm}$. Section 1 contains a brief account of various types of nanoporous materials, including track etched membranes, microfabricated membranes, and nanoporous membranes. In section 2, biomedical applications of nanoporous materials will be reviewed. In section 3, we will discuss surface modification techniques to improve biocompatibility and fouling resistance. 
Finally, key scientific challenges and future directions will be discussed in the concluding remarks.

\section{Types of nanoporous membranes}

Nanoporous membranes, due to a diverse set of materials, pore geometry and fabrication techniques, can be broadly classified as follows:

a) Type of material: Nanoporous membranes encompass a wide range of inorganic (metals, ceramics etc), organic (polymeric) and composite materials. For liquid phase separation, various types of nanoporpous oxides, typically alumina, titania, zirconia and silica, have been developed [8]. Recently, inorganic nanoporous materials with well ordered pores such as anodic alumina and nanoporous silica have gained considerable attention for use in biomedical applications. A majority of the commercial filtration and liquid phase separation applications use polymeric ultra or microfiltration membranes. Some of the typical polymeric nanoporous materials are Nafion, polycarbonate, polyethylene terephthalate, polysulfone etc. Another important class is composite membranes. Membranes containing two different materials have been developed in order to improve selectivity as well as stability. Such nanoporous materials have been prepared by combining, for example, a polymer with a ceramic [8].

b) Type of fabrication method: Many different synthesis methods exist to fabricate nanoporous materials. One of them is the anodization process, in which nanoporous films are prepared by selective electrochemical leaching of substances from solids. Nanoporous materials can be made with microfabrication techniques such as lithography as well as focused ion beam etching [9]. Two most common routes to nanoporous polymeric films are iontrack etching and phase separation technique [10,11]. Another widely used method is sol-gel process, which is often used to grow multi-component ceramic nanoporous materials [8].

c) Type of pores (size, shape and order): Porous materials are often distinguished based on pore size, size distribution, shape and order. Typically, they are classified as microporous $(<2 \mathrm{~nm})$, mesoporous $(2-50 \mathrm{~nm})$ and macroporous (> $50 \mathrm{~nm}$ ) based on size [7]. Porous materials are considered uniform if the size distribution is narrow as opposed to a wide pore-size distribution. The pores can be cylindrical, conical, slit-like, or irregular in shape. They can be well ordered with a vertical alignment as opposed to a random network of tortuous pores.

Table 1 summarizes various nanoporous membranes based on the type of material and the fabrication technique. Nanoporous materials that are important to biomedical applications will be summarized, with a special emphasis on materials with ordered pores.

The successful application of nanoporous interfaces in biomedical devices depends on several critical membrane properties (Fig. 1). First, the ability to fabricate membranes with a desired pore size and a narrow pore size distribution will enable a precise control over molecular transport. Second, in many applications a low flow resistance to enable high flux (characterized by high porosity and low membrane thickness) is desired [12]. Third, an adequate mechanical strength with respect to transmembrane pressure and sufficient thermal and chemical stability under a wide range of biological environment are essential for long term usage. Finally, for in vivo applications, biocompatibility and resistance to biofouling are vital to prevent immunological response and loss of functionality, respectively. 
At present, the majority of the applications involving biomolecular separation use polymeric membranes with a random pore matrix. For example, polymer gels (agarose or polyacrylamide) are routinely used in gel based electrophoresis for size separating DNA, proteins etc. Another technologically relevant class of polymer membranes is porous polymer matrices formed by initiating the precipitation of a polymer from solution. The solution precipitates into polymer rich domains that encompass voids to form a filter structure. The structure of pores in these materials is random, often characterized by tortuous pore paths and with broad pore size distributions. These polymer matrices can inexpensively separate biomolecules in large quantities by size (e.g., for sample preconcentration).

However, a well ordered pore structure is desired for controlled molecular transport and separation. In addition, a narrow pore size distribution with no tail to long pore sizes is a prerequisite to discriminating molecules with a small difference in size. While it is a challenge to fabricate thin, robust nanoporous membranes with very precise pore sizes and geometry, advances in nanofabrication methods have opened up exciting new avenues to overcome the limitations of conventional porous materials.

\subsection{Polymeric membranes}

Porous organic materials play an important role in biomedical applications and are under extensive investigation $[10,11,13]$. By far the most common method to fabricate polymeric porous membranes with relatively ordered pore arrays as well as pores close to cylindrical geometry is ion track technology. Filtration membranes are routinely manufactured from various polymers including polycarbonate and polyethylene terephthalate. The process involves irradiating a thin polymeric film with accelerated heavy ions, which leave so-called ion tracks. These ion tracks can then be enlarged to pores by chemical etching with an appropriate reagent that preferentially attacks the damaged track zone. Cylindrical or conical pores are produced with diameters in the range of $10 \mathrm{~nm}$ to micrometers $[10,11,13,14]$. These membranes have their limitations because the preparation of pores with diameters in the lower nanometer range is not possible.

Another very interesting technique to produce membranes is via the phase separation micromolding $(\mathrm{PS} \mu \mathrm{M})$ [11]. Phase separation micromolding $(\mathrm{PS} \mu \mathrm{M})$ is a replication technology based on phase separation, in which a polymer solution cast into a mold is solidified by phase separation and, during the solidification, assimilates the profile of the mold. The new developments in organic nanoporous structures in terms of structural and chemical properties and their applications are reviewed in detail in the papers by Ulbricht [11] as well as by Langley and Hulliger [15].

\subsection{Microfabricated membranes}

Recently, micro- and nanofabrication technology has emerged as an attractive way to make ordered and cylindrical arrays of nanopores on silicon and silicon nitride surfaces. Considerable effort has been devoted to developing porous silicon and silicon nitride membranes using lithography. In an attempt to overcome the problems such as broad pore size distributions, poor mechanical properties and biochemical instability associated with the conventional polymeric membranes, Desai et al. [16] proposed micromachined nanoporous biocapsules with well-controlled pore-sizes as small as $7 \mathrm{~nm}$ to provide immunoisolating microenvironments for encapsulated cells. These silicon nanoporous membranes are biocompatible, allow for the free exchange of nutrients, waste products and secreted therapeutic proteins and provide immunoisolation to transplanted cells. In addition, microfabricated porous silicon membranes have been applied to an implantable artificial pancreas [17] and oral drug delivery systems [18]. 
Membranes with very regular pore structures, pore sizes down to $100 \mathrm{~nm}$, and thickness values of 1-5 $\mu \mathrm{m}$ are often termed "nanosieves"; these structures can be achieved through photolithography and focused ion beam etching. For example, Tong et al. have fabricated an array of very uniform cylindrical nanopores with a pore diameter as small as $25 \mathrm{~nm}$ in an ultrathin micromachined silicon nitride membrane using focused ion beam (FIB) etching [19].

\subsection{Anodized membranes}

One of the extensively investigated nanoporous materials using electrochemical-etching technique is anodic aluminum oxide (AAO) membranes. Porous alumina membranes obtained with common anodic processes usually have a honeycomb-like pore structure with short distance ordering [20-22]. The pore geometry and morphology can be controlled by the conditions during the anodization processes. In recent years, a great success has been made in the preparation of AAO membranes with highly ordered nanochannel arrays. Conventional anodizing of aluminum could produce ordered nanochannel array with a limited range of pore-sizes through self-organized process [20, $23-25]$. The degree of the self-organization in the hole-array depends on the anode voltage as well as the acid used in fabrication. However, the most effective method for developing ordered porous anodic alumina with large dimension is the pretexture process, in which a shallow ordered pattern is first produced on the surface of an aluminum wafer, and the ordered channel structures are obtained by the following anodization process [23]. Using this method, Masuda and coworkers produced porous anodic alumina with almost defect-free, ordered channel arrays [26]. The size of the membrane was on the order of millimeters, with a channel density of $10^{10} \mathrm{~cm}^{-2}$ and an aspect ratio over 150 . The smallest channel diameter was about $70 \mathrm{~nm}$ with $100 \mathrm{~nm}$ intervals. Fig. 2 shows the channel-array architectures of the anodic alumina with different intervals obtained by Masuda et al. [26]. The anodization of aluminum was conducted in oxalic acid. Further investigation has indicated that the channel interval can be modified by controlling the pretextured-pattern interval or the applied voltage [27].

Recently, Lee et al. [28] have used a hard anodization process that enables fast fabrication of AAO membranes with pore sizes down to $40 \mathrm{~nm}$ making them ideal for size sorting a wide range of molecular sizes.

AAO membranes have been explored for use in diffusion controlled filtration systems. Kipke and Schmid [29] have demonstrated the suitability of alumina membranes for size sorting by studying the diffusion of crystal violet molecules. In particular, they showed that the diffusion of crystal violet molecules encapsulated inside micelles is determined by the pore size whereas the diffusion of uncoated molecules showed no pore size dependence. In a similar work, Gong et al. [30] have conducted molecular release experiments using nanoporous AAO capsules and demonstrated that the biocapsules successfully prevented diffusion of molecules larger than a cutoff size.

In addition to anodized alumina, electrochemical etching has been widely used to develop nanoporous semiconductors. Silicon was the first semiconductor material used to fabricate nanoporous structures with electrochemical-etching technique [31, 32]. More recently, nanoporous semiconductors have been developed with electrochemical etching of, for example, germanium $[33,34]$.

\subsection{Ordered nanoporous semiconductors}

Ordered and patterned silicon porous structures have been studied most extensively, encouraged by the applications such as MEMS and biomedical devices. Unlike anodized alumina, and other semiconductors, self-organized pore is difficult to be realized on silicon [31]. The processes of patterned nanoporous silicon often require the use of lithography, 
oxidization, and $\mathrm{Si}_{3} \mathrm{~N}_{4}$ deposition processes in a clean room environment. Létant and coworker prepared pores on pre-patterned n-type silicon wafers using the backside illumination; these materials were developed for selective bio-organism capture [35].

\section{Biomedical applications of nanoporous materials}

Many promising biomedical applications for nanoporous materials have been discovered and several are currently being explored. In implantable devices the membrane would function as a semipermeable compartment that holds the implant or drug while allowing passage of desired molecules in a controlled way. Nanoporous membranes are also suitable for a wide variety of biomolecular analysis applications. Possible in vitro applications include diagnosis and protein separation. Enormous research efforts in the past decade have been applied to automate biological analyses and to reduce sample consumption and cost. The efforts have led to the development of many microfabricated devices performing separation, mixing, reaction, detection, or preconcentration. In particular, extraction of analytes from complex samples has been achieved using variations in diffusivity and selective transport through membranes. There has been a great push towards the micro total analysis system ( $\mu$ TAS) or lab-on-a-chip concept [36]. In this section, a brief overview of biomedical applications involving nanoporous membranes is presented. While the applications are classified based on the functionality of the membrane, there is clearly some overlap between them. These applications are summarized with a schematic diagram in Fig. 3.

\subsection{Separation and sorting of biomolecules}

Separation and sorting is used for isolation and purification of molecules from various biological feed streams and is important in many fields including pharmaceutical industry, food industry, and biotechnology. Many techniques, including size exclusion chromatography and gel electrophoresis of biopolymers, are currently used in separation science $[36,37]$. Biomolecular separation in more ordered pores has recently been examined for various applications. In the kidney application, the synthetic nanoporous membrane acts as a support for kidney cells and as a blood filter that retains serum proteins while allowing smaller waste substances out [38].

The flow of materials through nanopores can also be externally regulated. For example, Martin and coworkers [39] have fabricated a membrane that consists of an array of cylindrical gold nanotubules with pore radius less than $1 \mathrm{~nm}$. They demonstrated that by switching charge on the tubules from positive to negative, ions of opposite charge could be rejected, allowing for voltage gating of oppositely charged molecules.

\subsection{Biosensing}

Sensory systems use a variety of membrane bound protein pores to detect molecules and facilitate cells to respond to stimuli. Such biosensing is also important in many technological areas including pharmaceutical industry, medical diagnosis and detection of hazardous biomolecules. In a majority of these applications the biosensing device combines a biological component with a physiochemical detection component to detect analytes in biological feed streams. For example, amperometric sensors utilize an immobilized enzyme for the conversion of the target analytes in a biological system into electrochemically detectable products. It is one of the most widely used detection methods for the determination of blood glucose. Glucose oxidase immobilized in porous nanocrystalline $\mathrm{TiO}_{2}$ film is shown to be capable of sensing blood glucose [40]. Similarly, cholesterol biosensors have been developed that rely sensing on immobilized cholesterol oxidase enzymes inside $\mathrm{ZnO}$ nanoporous thin films [41]. 
Also worth mentioning are the amperometric-type enzymeless nanoporous systems. For example, recently a glucose sensing system based on nanoporous platinum electrode embedded in a microfluidic chip comprised of a microfluidic transport channel network and a miniaturized electrochemical cell has been demonstrated [42]. Bohn and co-workers have proposed exploiting an array of electrically switchable nanocapillary membranes to perform sequential sensing and analytic operations in $\mu$ TAS devices [6]. Another type of biosensor is a nanoporous material that analyzes one molecule at a time [43]. A brief discussion of these important materials is provided in the following subsection.

\subsection{Single molecular analysis}

Nanoporous supports have gained prominence in probing biomacromolecules (DNA, RNA, and proteins) one by one for single-molecule analysis. It is now well established that by measuring the frequency, magnitude and duration of blockage in ion-current of an electrolyte when biomolecules are drawn through nanopores embedded in insulating membranes, it is possible to access information on the concentration, structure, size and sequence of , for example, single and double stranded DNA or RNA [44]. This technique has been applied in detecting biomolecules by using engineered membrane-bound receptors such as a-hemolysin (a-HL) protein pores embedded in a lipid membrane [45]. Much of the early research in single molecule analysis used lipid membranes incorporated inside micron sized apertures in polymeric films such as Teflon. One of the drawbacks of the polymeric supports with micron-sized pores is that the lipid membranes tend to rupture after a brief period of usage demanding techniques to improve the durability in order to be useful in practical applications. Nanoporous membranes have the potential to support protein pores for single molecule analysis [43].

The range of sensing applications of lipid-bound nanopores is limited by the architecture of the protein pores. Synthetic nanopores have been explored recently in an effort to expand the functionality of single molecule detectors. Synthetic nanopores using a variety of materials, such as glass, polymers and solid state membranes have been demonstrated to be useful for sensing single-stranded and double-stranded DNA, ions, macromolecules, and proteins. Synthetic nanopores fabricated with nanoscale control over pore size and pore surface chemistry would allow regulating analyte-surface interactions and thereby provide means to impart desired functionality to the sensor. Arrays of these sensor platforms with sensitivity for multiple analytes can be incorporated in microfluidic devices to create lab-on-a-chip technology [46].

\subsection{Immunoisolation}

Immunoisolation refers to protecting implanted cells or drug release systems from an immune reaction. It is usually accomplished by encapsulation using a nanoporous semipermeable membrane. The use of nanoporous membranes serves to isolate the transplanted cells from the body's immune system. These pores are large enough to allow small molecules such as oxygen, glucose, and insulin to pass, but are small enough to impede the passage of much larger immune system molecules such as immunoglobulin. For example, in the artificial pancreas, insulin secreting pancreatic cells are encapsulated inside an immunoisolating device and a semipermeable membrane acts as an interface between the cells and the body. Desai et al. have explored using nanoporous silicon interfaces prepared by microfabrication techniques in implantable artificial pancreas to treat diabetes [47]. It is important for the nanoporous membrane to be biocompatible and biofouling-resistant for many in vivo functions. 


\subsection{Drug delivery}

The development of in vivo delivery systems to enable supply of drugs locally where they are needed in a controlled manner is actively sought after. The purpose of controlled delivery systems is to administer drugs in a chosen way for more effective therapy and to eliminate the possibility of improper dosing. Nanoporous membranes with well-controlled pore size, porosity and membrane thickness offer an attractive route for making capsules that may be used for provide controlled release of pharmacologic agents [48]. For example, nanoporous materials are researched for use in drug eluting stents to treat coronary artery disease [17]. Recently, nanoporous inorganic membranes have been tested for sustained release of ophthalmic drugs to treat conditions related to the eye [3]. When coupled to biosensors, smart drug delivery systems that respond to physiological conditions could be developed.

\section{Surface modifications to improve membrane properties}

When biomedical implants come in contact with physiological environments, primarily three types of reactions limit the long term usage. These are (i) biofouling, (ii) immune reaction as determined by biocompatibility and (iii) degradation and loss of implant material due to corrosion, dissolution or leaching. Several different approaches are being explored to address these issues such that the future medical implants interact with the host in a controlled and a predictable manner.

Biofouling is the accumulation of cells, proteins, and other materials from the biological environment on a medical device surface in a way that diminishes or prevents medical device function. It is caused by adsorptive and adhesive interactions of small molecules, proteins, and cells on the surface. The biofouling process starts immediately upon contact of a synthetic material with the body. The adsorbed proteins prevent diffusion of small molecules to and from an active medical device. Another problem is tissue encapsulation which is characterized by fibroblast proliferation, collagen synthesis, blood vessel proliferation, and other cellular processes. These events lead to the formation of a vascular connective tissue capsule, which retards the transport of low-molecular weight molecules (e.g., glucose) due to increased diffusion path tortuosity and steric hindrance.

Poor biocompatibility of medical device packaging can also lead to device failures. The biocompatibility of an implantable device is dictated by the inflammatory response that occurs after device implantation. Wound healing consists of several stages, including hemostasis, inflammation, repair, and scar formation. During device implantation, connective tissue cells and epithelial cells are destroyed and the basement membrane is disrupted. This injury initiates the inflammatory response responsible for wound healing. Soon the injured region contains clotted blood. This fibrin clots attract neutrophils within one day after injury, these inflammatory cells affect the device function by their simple presence, as they consume nutrients and release proteolytic enzymes and free radicals within the vicinity of the implant. By day 3, macrophages and granulation tissue are common features. By day 5 , neovascularization is observed at the wound site. Epithelium migrates and collagen fibrils begin to bridge tears in the implant site. At one week, inflammatory features have essentially ceased, although there is still connective tissue proliferation. Finally, by the end of the first month, a mature scar capsule is completed within the epithelial layer. The biocompatibility of the sensor surface material will influence the characteristics of each stage in this process. The inflammatory tissue response limits active device functionality. For example, a thick, avascular, and fibrous implant scar capsule leads to reduced analyte diffusion and uptake, resulting in a diminished sensor response. 
Several investigators have examined the use of coatings and other modified surfaces in order to reduce the biofouling of sensors. A good review on this topic can be found in reference [1]. Hydrogels were one of the first materials used to mitigate biofouling of active medical devices. The most commonly used hydrogels are the cross-linked polymers of either poly(hydroxyethyl methacrylate) (PHEMA) or poly(ethylene glycol) (PEG). Coatings of these polymers are flexible, polar, uncharged and water swellable. These materials produce a hydrophilic interface between the solid surface and aqueous bulk, thereby masking the surface. Both PHEMA and PEG are appealing coatings for sensors because water-soluble analytes can diffuse through the water-swollen polymer layer. The cross-link density of the gel controls the amount of analyte diffusion.

Several problems have been encountered in applying hydrogels to the device surface: (1) hydrogels exhibit poor adhesion to substrates; (2) hydrogels have poor mechanical stability and are unable to withstand the forces of implantation; (3) the monomer, solvent and crosslinking agent may have detrimental effects on the biological components of the biosensor; and (4) hydrogels may serve to limit analyte diffusion into the sensor. Hydrogel coatings are not a viable solution to the biofouling problem.

Another strategy for optimizing the active medical surface is to cover it with a coating that mimics the body [ $49-51]$. The surface should ideally act like a cell membrane, selectively and specifically binding certain molecules while resisting undesirable, non-specific interactions. Major research efforts have been undertaken to characterize phospholipid, phospholipid-containing, or phospholipid-like biomaterials. Unfortunately, phospholipid membranes are fragile and thus difficult to deposit; hence, most attempts have been aimed at developing phospholipid-modified polymers. Phospholipid-modified polymer coatings tested in vivo showed significant reductions in platelet adhesion, platelet activation, and fibrinogen adsorption. Ultimately, these materials represent a promising, method to prevent biofouling over relatively short periods of time.

Nafion is a perfluorosulfonic acid polymer that has been studied for use as a coating for several active medical devices. Nafion is a chemically inert, anionic polymer with both hydrophobic and hydrophilic properties. Nafion films applied at a thickness of $1-2 \mu \mathrm{m}$ promote a low amount of adsorption of molecules from solutions. Unfortunately, these coatings are chemically stable only for a few weeks; cracking, flaking, protein adhesion, and calcium deposition are frequent problems.

A surfactant ("surface active agent") is a molecule that seeks the interface between two phases [52]. Surfactant molecules possess both hydrophobic and hydrophilic character. Surfactants are usually lipids with polar (e.g.,anionic, cationic, or uncharged) head groups attached to hydrocarbon tails. Surfaces that have been adsorbed with pluronic ${ }^{\circledR}$ surfactants (PEO-PPO-PEO triblock copolymers) possess resistance to protein adsorption and cell adhesion. Many membranes used in electrochemical sensors contain these surfactant plasticizing molecules. Unfortunately, the surfactants continually diffuse to the surface and out of the membrane until they are depleted. Consequently, these surfactants need leaching antifouling agents, such as calcium chelating plasticizers that limit Factor X activation (an enzyme in the coagulation cascade), in order to continuously renew the sensor surface. However, Lindner et al. recently demonstrated that plasticizers leaching from implanted sensor membranes can cause tissue inflammation. As such, the lifetime of a surfactantcoated device is seriously limited [53].

Regrettably, none of the biosensor packaging techniques described above entirely eliminates biofouling. Experiments have shown these surface treatments undergo mineralization in the biological environment; these processes result in cracking and altered device permeability. 
The inflammatory process described above is not eliminated, it is shifted from seconds or minutes post-implantation to tens of minutes or days post-implantation.

There are solutions to many of these problems on the horizon. For example, chemical grafting of PEG chains has attracted much attention as a way to develop nonfouling interfaces. PEG grafted surfaces have demonstrated a low protein, cell and bacterial adhesion due to its favorable interaction with water. The grafted PEG chains form a polymer brush layer and thus exclude other molecules due to repulsive interaction and hence resist protein adsorption [54]. Further, there is evidence that PEG coating can also improve biocompatibility of the implants. For example, in vivo tests have demonstrated that while implantation of AAO capsules into the peritoneal cavity of rats induces a transient inflammatory response, poly(ethylene glycol) coatings are useful in minimizing the host response to the material [55].

Diamond-like carbon thin films are another suitable material for implantable biosensors. The term diamond-like carbon is used to describe amorphous carbon that contains $\mathrm{sp}^{3}$ hybridized carbon atoms. In a recent study, Narayan et al. demonstrated that diamondlike carbon-coated nanoporous alumina membranes remained free from fibrin or platelet aggregation after exposure to human platelet rich plasma [56]. Figure 4 shows that no fibrin networks or platelet aggregation were observed on the surface of the diamond-like carbon-coated nanoporous membrane ( $100 \mathrm{~nm}$ pore size) after exposure to human platelet rich plasma. Only a few small, widely scattered sodium chloride crystals were observed on the film surface. On the other hand, uncoated membranes exhibited significant protein adsorption and pore fouling. The clotting process has been attributed to a charge transfer theory first proposed by Srinivasan et al., in which an exchange of electrons between blood proteins and the surface of a material initiates the release of fibrinopeptides that bring about thrombosis [57]. The charge transfer rate during platelet rich plasma-material interaction is a function of the time constant of the material. The time constant obtained for diamond-like carbon is higher than those obtained for thrombogenic metal-containing materials. As a result, the diamondl-ike carbon coatings allow a lower amount of charge transfer. Studies have shown that platelet adhesion occurs less often on diamond-like carbon surfaces than on titanium, titanium nitride, and stainless steel surfaces [58-60]. Jones et al. have suggested that the low surface energy values $\left(40-44 \mathrm{mN} \mathrm{m}^{-1}\right)$ and large contact angle values $\left(75-80^{\circ}\right.$ for water) exhibited by diamond-like carbon limit protein fouling [61].

The biological properties of uncoated nanoporous alumina membranes as well as nanoporous alumina membranes coated with gold, silicon, and diamond-like carbon thin films were examined using MTT viability assay. Silicon (Si), gold (Au), diamond-like carbon (DLC) coatings were deposited on nanoporous alumina membranes using ultraviolet (wavelength $=248 \mathrm{~nm}$ ) pulsed laser deposition, which is a physical vapor deposition process. Figure 5 depicts a comparison of MTT viability for gold-coated, silicon-coated, diamond-like carbon-coated, and uncoated nanoporous alumina membranes (control), which shows a significant decrease in human epidermal keratinocyte (HEK) viability with the addition of gold-coated membranes or silicon-coated membranes in comparison to diamondlike carbon coated membranes or uncoated alumina (Al) membranes. However, materials such as gold are considered to have a minimal effect on cells [62].

Coating such materials might also improve chemical stability and leaching resistance of nanoporous membranes under physiological conditions. Perhaps a more efficient method to modify nanoporous membranes is by atomic layer deposition (ALD). ALD is a thin film growth technique that utilizes alternating, self-limiting chemical reactions between gaseous precursor molecules and a surface to deposit material in an atomic layer-by-layer fashion [63]. Saturation of the individual reactions ensures that all surfaces of a substrate are coated 
with exactly the same thickness, even for surfaces that are deeply embedded within a porous material. Consequently, ALD can apply precise layers onto surfaces with extremely high aspect ratio features such as the nanopores in membranes and thus allowing for conformal coating [64]. Thus the upshot of the technique is that it leaves no unexposed area unlike in line-of-sight coating techniques which is critical when the bulk of the membrane is to be shielded from the harsh biological fluids. A wide variety of materials can be deposited using ALD including oxides, nitrides, and metals. There is evidence that ALD coating of AAO with Pt and subsequent functionalization with PEG improves resistance to leaching and reduces fouling [12]. Besides the potential to impart desired surface chemistry, this technique provides a conformal coating of nanoporous membranes to achieve a very precise pore size control, shrinking down their diameters to below ten nanometers with a narrow distribution. For example, tailor-made compositions and pore sizes have been prepared by depositing thin films on AAO membranes using ALD [65].

With the general nature of the medical device and the biological environment of the implant's location in mind we have to address the biocompatibility of the interface to minimize biofouling, chemical degradation and leaching as well as inflammatory response $[66,67]$. The choice of an ideal surface coating will depend on the problem at hand. It is also worth pointing out that biofouling and leaching can be problems in in vitro applications as well.

\section{Conclusion}

Nanoporous materials are crucial to many biomedical applications such as immunoisolation devices, dialysis, smart as well as targeted drug delivery systems, bioanalytical devices and biosensors. Some of the key properties that these membranes are required to possess are a pore size of a few tens of a nanometer or less, a narrow pore size distribution to ensure higher selectivity, and a high porosity and low thickness to enable high flux, mechanical and chemical stability. Existing commercial membranes offer inexpensive means to separate bioanalytical applications such as large-scale preconcentration. On the other hand, development of truly nanoscale devices calls for more controlled pore architecture and the ability to sort and dispense molecules very precisely. The effort to find suitable nanoporous interfaces for the diverse biomedical applications will rely on an interdisciplinary approach involving materials science, chemistry, biology and engineering. As discussed in this paper, advanced nanofabrication methods have enhanced our abilities to fabricate well-ordered monodisperse nanopores in ultrathin membranes. Another promising possibility of nanofabrication is the prospect of integrating nanoporous membranes in lab-on-a-chip microfluidic systems for medical diagnostics.

In addition to control over pore geometry, biocompatibility and biofouling are central issues when membranes are used as interfaces in implantable devices. Many challenges lie in engineering biocompatible and nonfouling nanoporous supports for in vivo drug delivery applications. Surface modification by coating nanoporous membranes with inorganic as well as organic materials is currently being explored. While techniques such as modification with grafted polymers and self-assembled polymers have given promising results during in vitro testing, in vivo testing will be conducted in the future.

Looking into the future, the next generation nanoporous materials can be envisioned with multiple functionalities such as size screening, responsive flow regulation, dynamic pore sizing using external controls etc. Several approaches as described in this paper are currently being explored in research laboratories to impart stimuli-responsive gating ability to nanoporous membranes [12]. An example for such a smart membrane is pore surface modification with responsive polymers that undergo a conformational transition in response to stimuli such as $\mathrm{pH}[68,69]$, temperature [70] and ion concentration [71]. A significant 
challenge is to construct composite nanoporous membranes that respond to multiple stimuli. Such novel systems can be used in nano/micro scale chips for programmable drug delivery and biomolecular analysis.

\section{Acknowledgments}

This work is supported in part by the US Department of Energy's Office of Basic Energy Sciences, under contract no. DE-AC02-06CH11357. Also, support from the National Institute of Biomedical Imaging and BioEngineering (5R21EB003090-02) is acknowledged.

\section{References}

[1]. Wisniewski N, Reichert M. Methods for reducing biosensor membrane biofouling. Colloids and Surfaces B: Biointerfaces. Oct; 2000 18(11-12):197-219.

[2]. Andrade JD, Hlady V. Protein adsorption and materials biocompatibility - A tutorial review and suggested hypotheses. Adv. Poly. Sci. Jan.1986 79:1-63.

[3]. Orosz KE, Gupta S, Hassink M, Abdel-Rahman M, Moldovan L, Davidorf FH, Moldovan NI. Delivery of Antiangiogenic and Antioxidant Drugs of Ophthalmic Interest through a Nanoporous Inorganic Filter. Mol. Vis. Aug.2004 10:555-565. [PubMed: 15332016]

[4]. Lioni L, Boiarski A, Desai TA. Characterization of Nanoporous Membranes for Immunoisolation: Diffusion Properties and Tissue Effects. Biomed. Microdev. May; 2002 4(2):131-139.

[5]. Huang Z, Zhang W, Yu J, Gao D. Nanoporous Alumina Membranes for Enhancing Hemodialysis. Journal of Medical Devices. Mar; 2007 1(1):79-83.

[6]. Gatimu EN, Sweedler JV, Bohn PW. Nanofluidics and the Role of Nanocapillary Array Membranes in Mass-Limited Chemical Analysis. Analyst. Jun.2006 131:705-709. [PubMed: 16732357]

[7]. Liu, GQ.; Zhao, XS. Nanoporous Materials: Science and Engineering. Imperial College Press; London: 2003.

[8]. Tsuru T. Inorganic Porous Membranes for Liquid Phase Separation. Sep. Pur. Methods. Jul; 2001 30(2):191-200.

[9]. Kuiper S, van Rijn CJM, Nijdam W, Elwenspoek M. Development and applications of very high flux microfiltration membranes. J. Membr Sci. Nov; 1998 150(1):1-8.

[10]. Trautmann C, Brüchle W, Sphor R, Vetter J, Angert N. Pore geometry of etched ion tracks in polyimide. Nucl. Instr. Meth. B. Apr.1996 111:70-74.

[11]. Ulbricht M. Advanced functional polymer membranes. Polymer. Mar.2006 47:2217-2262.

[12]. Adiga SP, Curtiss LA, Elam JW, Pellin MJ, Shih CC, Shih CM, Lin SJ, Su YY, Gittard SD, Zhang J. Nanoporous materials for biomedical devices. JOM. Mar; 2008 60(3):26-32.

[13]. Metz S, Trautmann C, Bertsch A, Renaud Ph. Polyimide microfluidic devices with integrated nanoporous filtration areas manufactured by micromachining and ion track technology. J. Micromech. Microeng. Mar; 2004 14(3):324-331.

[14]. Sexton LT, Horne LP, Martin CR. Developing synthetic conical nanopores for biosensing applications. Mol. BioSyst. Oct.2007 3:667-685. [PubMed: 17882330]

[15]. Langley PJ, Hulliger J. Nanoporous and mesoporous organic structures: new opening for materials research. Chem. Soc. Rev. 1999; 28(5):279-191.

[16]. Desai TA, West T, Cohen M, Boiarski T, Rampersaud A. Nanoporous microsystems for islet cell replacement. Adv. Drug Del. Rev. Sep; 2004 56(11):1661-1673.

[17]. Desai TA, Chu WH, Tu JK, Beattie GM, Hayek A, Ferrari M. Microfabricated Immunoisolating Biocapsules. Biotech. and Bioeng. Jan; 1998 57(1):118-120.

[18]. Tao SL, Desai TA. Microfabricated drug delivery systems: from particles to pores. Adv. Drug Delivery Rev. Feb; 2003 55(3):315-328.

[19]. Tong HD, Jansen HV, Gadgil VJ, Bostan CG, Berenschot E, van Rijn CJM, Elwenspoek M. Silicon Nitride Nanosieve Membrane, Nano Lett. Feb; 2004 4(2):283-287.

[20]. O'Sullivan JP, Wood GC. The Morphology and Mechanism of Formation of Porous Anodic Films on Aluminium. Proc. Royal Soc. Lon. Series A. Jul.1970 317:511-543. 
[21]. Mardilovich P, Govyadinov AN, Mukhurov NI, Rzhevskii AM, Paterson R. New and modified anodic alumina membranes. Part I. Thermotreatment of anodic alumina membranes. J. Membr. Sci. Jan; 1995 98(1):131-142.

[22]. Mardilovich P, Govyadinov AN, Mukhurov NI, Paterson R. New and modified anodic alumina membranes. Part II. Comparison of solubility of amorphous (normal) and polycrystalline anodic alumina membranes. J. Membr. Sci. Jan; 1995 98(1):143-155.

[23]. Masuda H, Fukuda K. Ordered Metal Nanohole Arrays Made by a Two-Step Replication of Honeycomb Structures of Anodic Alumina. Science. Jun.1995 268:1466-1468. [PubMed: 17843666]

[24]. Masuda H, Hasegwa F, Ono S. Self-Ordering of Cell Arrangement of Anodic Porous Alumina Formed in Sulfuric Acid Solution. J. Electrochem. Soc. May; 1997 144(5):L127-L130.

[25]. Shingubara S, Morimoto K, Sakaue H, Takahagi T. Self-Organization of a Porous Alumina Nanohole Array Using a Sulfuric/Oxalic Acid Mixture as Electrolyte. Electrochem. Solid-State Lett. Jan; 2004 7(3):E15-E17.

[26]. Masuda H, Yamada H, Satoh M, Asoh H, Nakao M, Tamamura T. Highly ordered nanochannelarray architecture in anodic alumina. Appl. Phys. Lett. Nov; 1997 71(19):2770-2772.

[27]. Asoh H, Nishio K, Nakao M, Tamamura T, Masuda H. Conditions for Fabrication of Ideally Ordered Anodic Porous Alumina Using Pretextured Al. J. Electrochem. Soc. Apr.2001 148:B152-B156.

[28]. Lee W, Ji R, Gösele U, Nielsch K. Fast fabrication of long-range ordered porous alumina membranes by hard anodization. Nat. Mater. Sep.2006 5:741-747. [PubMed: 16921361]

[29]. Kipke S, Schmid G. Nanoporous Alumina Membranes as Diffusion Controlling Systems. Adv. Func. Mat. Dec; 2004 14(12):1184-1188.

[30]. Gong D, Yadavalli V, Paulose M, Pishko M, Grimes CA. Controlled Molecular Release Using Nanoporous Alumina Capsules. Biomed. Microdev. Mar; 2003 5(1):75-80.

[31]. Föll H, Christophersen M, Carstensen J, Hasse G. Formation and application of porous silicon. Mat. Sci. Eng. R: Reports. Nov; 2002 39(4):93-141.

[32]. Föll H, Carstensen J, Frey S. Porous and Nanoporous Semiconductors and Emerging Applications. Mater. Res. Soc. Symp. Proc. 2005; 876E:R12.1.1-13.

[33]. Langa S, Christophersen M, Carstensen J, Tiginyanu IM, Föll H. Electrochemical pore etching in Ge. Phys. Stat. Sol. A. 2003; 195:R4-6.

[34]. Fang C, Föll H, Carstensen J, Langa S. Electrochemical Pore Etching in Ge - an Overview. Phys. Stat. Sol. A. May.2007 204:1292-1296.

[35]. Létant SE, Hart BR, van Buuren T, Terminello LJ. Functionalized silicon membranes for selective bio-organism capture. Nature Mater. Jun.2003 2:391-395. [PubMed: 12717430]

[36]. Han, J. Introduction to Nanoscale Science and Technology. Di Ventra, M.; Evoy, S.; Heflin, JR., editors. Springer; 2004.

[37]. Ghosh, R. Protein Bioseparation Using Ultrafiltration: Theory, Applications and New Developments. Imperial College Press; London: 2002.

[38]. Fissell WH, Humesa HD, Fleischmanb AJ, Roy S. Dialysis and Nanotechnology: Now, 10 Years, or Never? Blood Purifi. Jan; 2007 25(1):12-17.

[39]. Nishizawa M, Menon VP, Martin CR. Metal Nanotubule Membranes with Electrochemically Switchable Ion-Transport Selectivity. Science. May.1995 268:700-702. [PubMed: 17832383]

[40]. Li Q, Luo G, Feng J, Zhou Q, Zhang L, Zu Y. Amperometric Detection of Glucose with Glucose Oxidase Absorbed on Porous Nanocrystalline TiO2 Film. Electroanalysis. Apr; 2001 13(5):413416.

[41]. Singh SP, Arya SK, Pandey P, Malhotra BD, Saha S, Sreenivas K, Gupta V. Cholesterol Biosensor Based on RF Sputtered Zinc Oxide Nanoporous Thin Film. App. Phys. Lett. Aug.2007 91:063901, 1-3.

[42]. Joo S, Park S, Chung TD, Kim HC. Integration of a Nanoporous Platinum Thin Film into a Microfluidic System for Non-enzymatic Electrochemical Glucose Sensing. Anal. Sci. Mar.2007 23:277-281. [PubMed: 17372368] 
[43]. Bayley H, Cremer BS. Stochastic Sensors Inspired by Biology. Nature. Sep.2001 413:226-230. [PubMed: 11557992]

[44]. Akeson M, Branton D, Kasianowicz JJ, Brandin E, Deamer DW. Microsecond Time-Scale Discrimination among Polycytidylic Acid, Polyadenylic Acid, And Polyuridylic Acid as Homopolymers or as Segments within Single RNA Molecules. Biophys. J. Dec; 1999 77(6): 3227-33. [PubMed: 10585944]

[45]. Kasianowicz JJ, Brandin E, Branton D, Deamer DW. Characterization of Individual Polynucleotide Molecules Using a Membrane Channel. Proc. Natl. Acad. Sci. Nov 26; 1996 93(24):13770-13773. [PubMed: 8943010]

[46]. Kim MJ, Wanunu M, Bell DC, Meller A. Rapid Fabrication of Uniformly Sized Nanopores and Nanopore Arrays for Parallel DNA Analysis. Adv. Mater. Dec.2006 18:3149-3153.

[47]. Tsujino I, Ako J, Honda Y, Fitzgera PJ. Drug Delivery Via Nano-, Micro and Macroporous Coronary Stent Surfaces. Expert Opinion on Drug Delivery. 2007; 4(3):287-295. [PubMed: 17489655]

[48]. Desai, TA.; Sharma, S.; Walczak, RJ.; Boiarski, A.; Cohen, M.; Shapiro, J.; West, T.; Melnik, K.; Cosentino, C.; Sinha, PM.; Ferrari, M. Nanoporous Implants for Controlled Drug Delivery. In: Desai, TA.; Bhatia, S., editors. BioMEMS and Biomedical Nanotechnology Volume III Therapeutic Micro/Nanotechnology. Springer; US:

[49]. Lewis AL. Phosphorylcholine-Based Polymers and their use in the Prevention of Biofouling. Colloids and Surfaces B: Biointerfaces. Oct; 2000 18(3-4):261-275.

[50]. Ishihara K, Nomura H, Mihara T, Kurita K, Iwasaki Y, Nakabayashi N. Why Do Phospholipid Polymers Reduce Protein Adsorption? J. Biomat. Res. Feb.1998 39:323-330.

[51]. Harrison DJ, Turner RFB, Baltes HP. Characterization of Perfluorosulfonic Acid Polymer Coated Enzyme Electrodes and a Miniaturized Integrated Potentiostat for Glucose Analysis In Whole Blood. Anal. Chem. Oct.1988 60:2002-2007. [PubMed: 3239784]

[52]. Lee JH, Kopecek J, Andrade JD. Protein-Resistant Surfaces Prepared By PEO-Containing Block Copolymer Surfactants. J. Biomed. Mater. Res. 1989; 23:351-368. [PubMed: 2715159]

[53]. Lindner E, Cosofret VV, Ufer S, Buck RP, Kao WJ, Neuman MR, Anderson JM. Ion-Selective Membranes with Low Plasticizer Content: Electroanalytical Characterization and Biocompatibility Studies. J. Biomed. Mater. Res. May; 1994 28(5):591-801. [PubMed: 8027099]

[54]. La Flammea KE, Popatb KC, Leonic L, Markiewiczc E, La Tempad TJ, Romanc BB, Grimesd CA, Desai TA. Biocompatibility of Nanoporous Alumina Membranes for Immunoisolation. Biomat. Jun; 2007 28(16):2638-2645.

[55]. Morra M. On the Molecular Basis of Fouling Resistance. J. Biomater. Sci. Polymer Ed. 2000; 11(6):547-569.

[56]. Narayan RJ, Jin C, Menegazzo N, Mizaikoff B, Gerhardt RA, Andara M, Agarwal A, Shih C, Shih C, Lin S, Su Y. Nanoporous Hard Carbon Membranes for Medical Applications. J. Nanosci. Nanotech. Apr.2007 7:1486-2493.

[57]. Srinivasan S, Sawyer PN. Role of Surface Charge of The Blood Vessel Wall, Blood Cells, and Prosthetic Materials in Intravascular Thrombosis. J. Colloid Interface Sci. Mar.1970 32:456-463. [PubMed: 5417531]

[58]. Monties JR, Dion I, Havlik P, Rouais F, Trinkl J, Baquey C. Cora Rotary Pump for Implantable Left Ventricular Assist Device: Biomaterial Aspects. Artif. Org. Jul.1997 21:730-734.

[59]. Jones MI, McColl IR, Grant DM, Parker KG, Parker TL. Protein Adsorption and Platelet Attachment and Activation on TiN, TiC, And DLC Coatings on Titanium for Cardiovascular Applications. J. Biomed. Mater. Res. Nov; 2000 52(2):413-421. [PubMed: 10951383]

[60]. Jones MI, McColl IR, Grant DM, Parker KG, Parker TL. Haemocompatibility of DLC and TiCTiN Interlayers on Titanium. Diam. Rel. Mater. Mar.1999 8:457-462.

[61]. Yu LJ, Wang X, Wang XH, Liu XH. Haemocompatibility of Tetrahedral Amorphous Carbon Films. Surf. Coat. Tech. Jun.2000 128:484-488.

[62]. Ritala, M.; Leskela, M. Atomic Layer Deposition. In: Nalwa, HS., editor. Handbook of Thin Film Materials. Vol. Vol. 1. Academic Press; San Diego: 2001.

[63]. Pistorius A, Willershausen B. Biocompatibility of dental materials in two human cell lines Europ. J. Med. Res. 2002; 7:81-88. 
[64]. Elam JW, Routkevitch D, Mardilovich PP, George SM. Conformal Coating on Ultrahigh-AspectRatio Nanopores of Anodic Alumina by Atomic Layer. Chem. Mater. 2003; 15(18):3507-3517.

[65]. Xiong G, Elam JW, Feng H, Han CY, Wang HH, Iton LE, Curtiss LA, Pellin MJ, Kung M, Kung H, Stair PC. Effect of Atomic Layer Deposition Coatings on the Surface Structure of Anodic Aluminum Oxide Membranes. J. Phys. Chem. B. Jul.2005 109:14059-14063. [PubMed: 16852765]

[66]. Ratner, BD.; Hoffman, AS.; Schoen, FJ.; Lemons, JE., editors. An Introduction to Materials in Medicine. Second Edition. Academic Press; San Diego: 2004. Biomaterials Science.

[67]. Park, JB.; Bronzino, JD., editors. Biomaterials: Principles and Application. CRC Press; Boca Raton, FL: 2002.

[68]. Mika M, Childs RF, Dickson JM. Chemical valves based on poly(4-vinylpyridine)-filled microporous membranes. J. Memb. Sci. 1999; 153(1):45-56.

[69]. Adiga SP, Brenner DW. Toward Designing Smart Nanovalves: Modeling of Flow Control through Nanopores via the Helix-Coil Transition of Grafted Polypeptide Chains. Macromol. 2007; 40(4):1342-1348.

[70]. Åkerman S, Viinikka P, Svarfvar B, Putkonen K, Järvinen K, Kontturi K, Näsman J, Urtti A, Paronen P. Drug permeation through a temperature-sensitive poly( $\mathrm{N}$ - isopropylacrylamide) grafted poly(vinylidene fluoride) membrane. Int. J. Pharma. 1998; 164:29-36.

[71]. Ito Y, Park YS. Signal-responsive gating of porous membranes by polymer brushes. Poly. Adv. Tech. 2000; 11(3):136-144. 


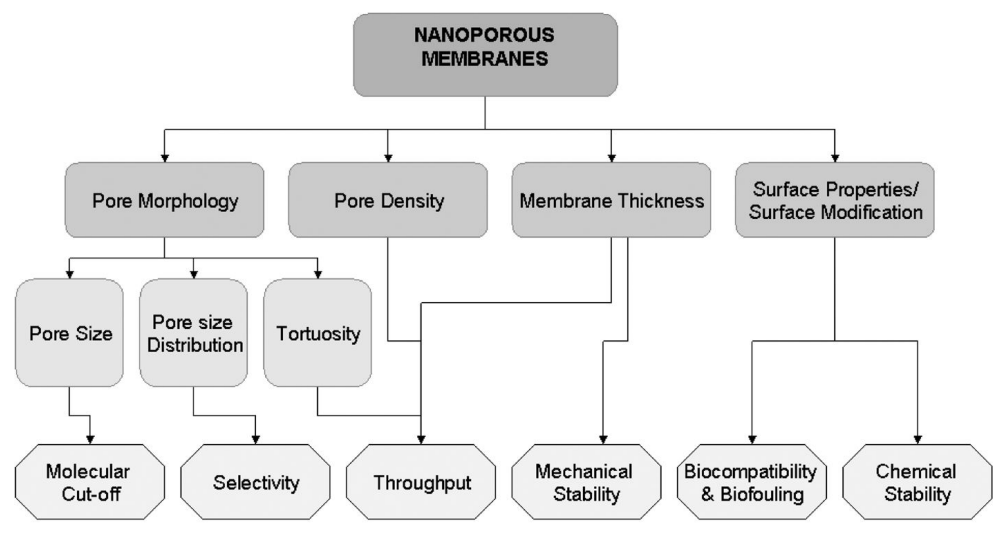

Figure 1.

A schematic diagram of key membrane characteristics that affect the performance. 


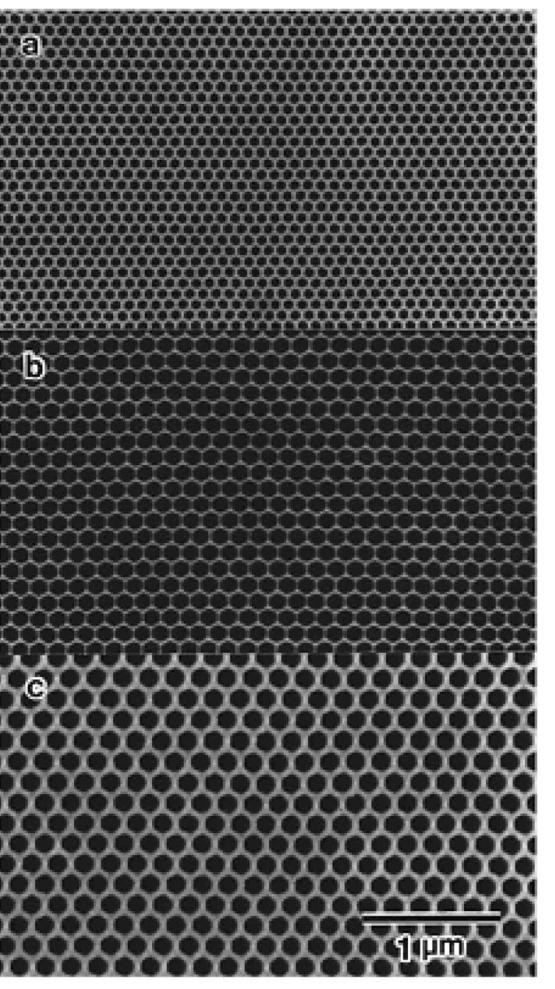

Figure 2.

SEM image of ordered porous structures of alumina. The intervals of $100 \mathrm{~nm}$ (a), $150 \mathrm{~nm}$ (b) and $200 \mathrm{~nm}$ (c). Reused with permission from Hideki Masuda, Applied Physics Letters, 71, 2770 (1997). Copyright 1997, American Institute of Physics. 


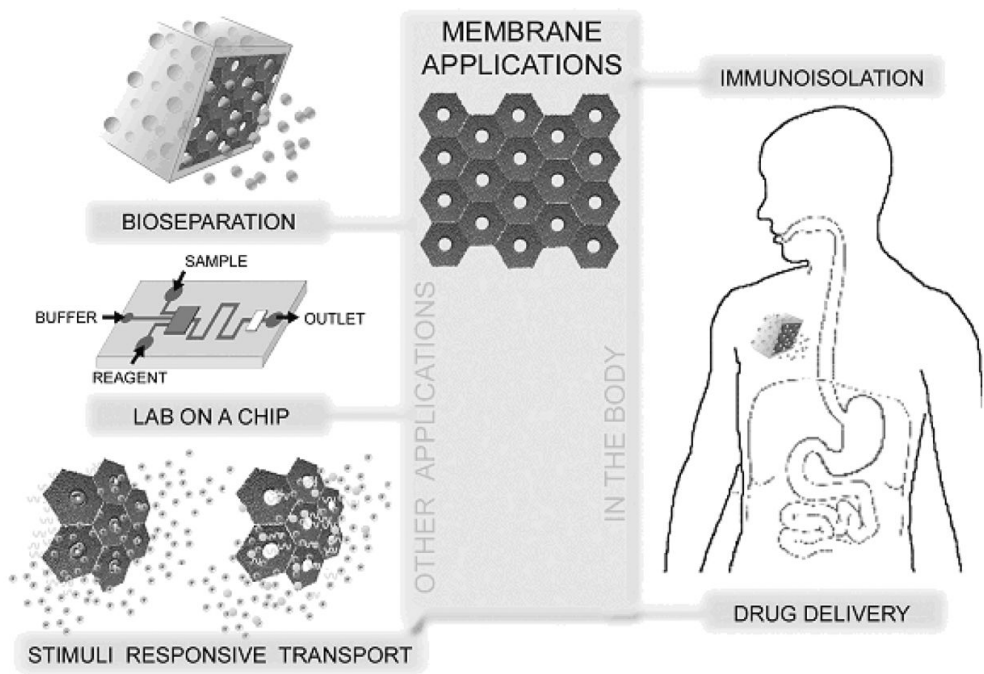

Figure 3.

Biological applications of nanoporous materials. 

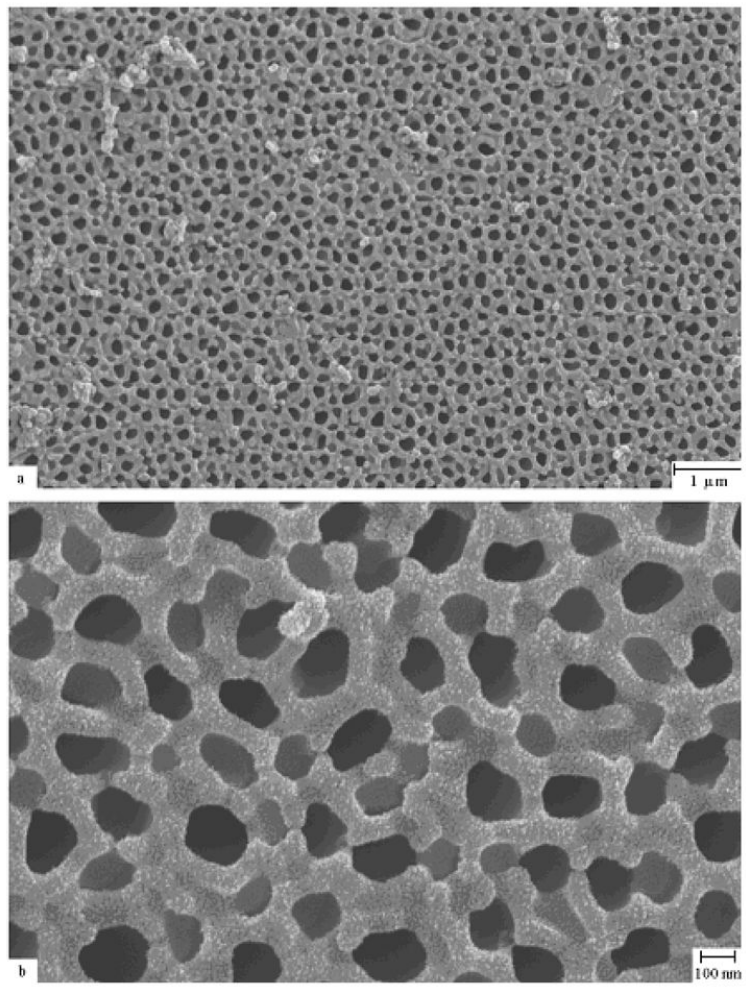

Figure 4.

Scanning electron micrograph of anodized diamond-like carbon-coated alumina membrane exposed to platelet rich plasma. The pulsed laser deposition method was used for the coating. The surface contains sodium chloride crystals; however, the pores remain free of fouling. Reprinted with permission from R. Narayan et al., Journal of Nanoscience and Nanotechnology, 7, 1486 (2007) Copyright @ American Scientific Publishers, http:// www.aspbs.com. 


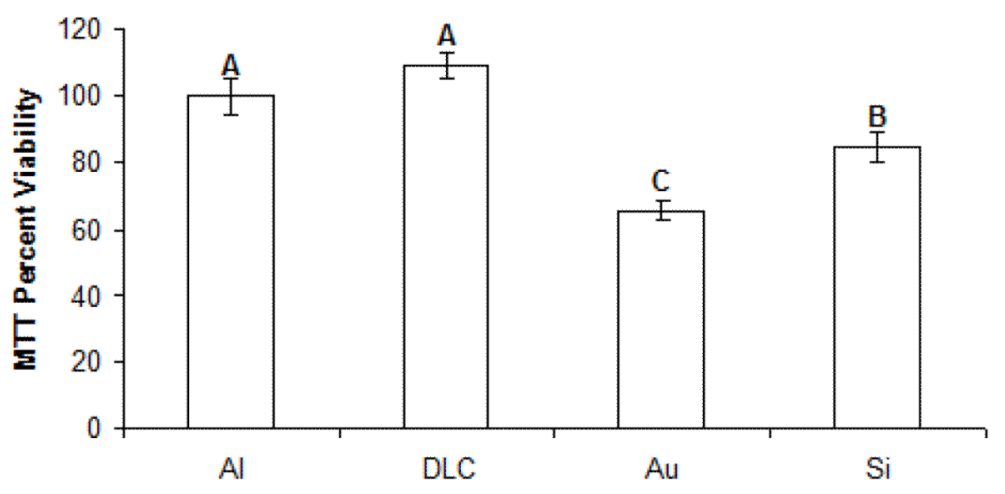

Figure 5.

Twenty-four hour MTT viability assays conducted in human epidermal keratinocytes for uncoated (Al), gold-coated (Au), silicon-coated ( $\mathrm{Si}$ ), and diamond-like-carbon (DLC)-coated nanoporous alumina membranes. $\mathrm{Si}, \mathrm{Au}$, and DLC coatings were deposited on nanoporous alumina membranes using ultraviolet (wavelength $=248 \mathrm{~nm}$ ) pulsed laser deposition. 
Table 1

Classification of nanoporous membranes.

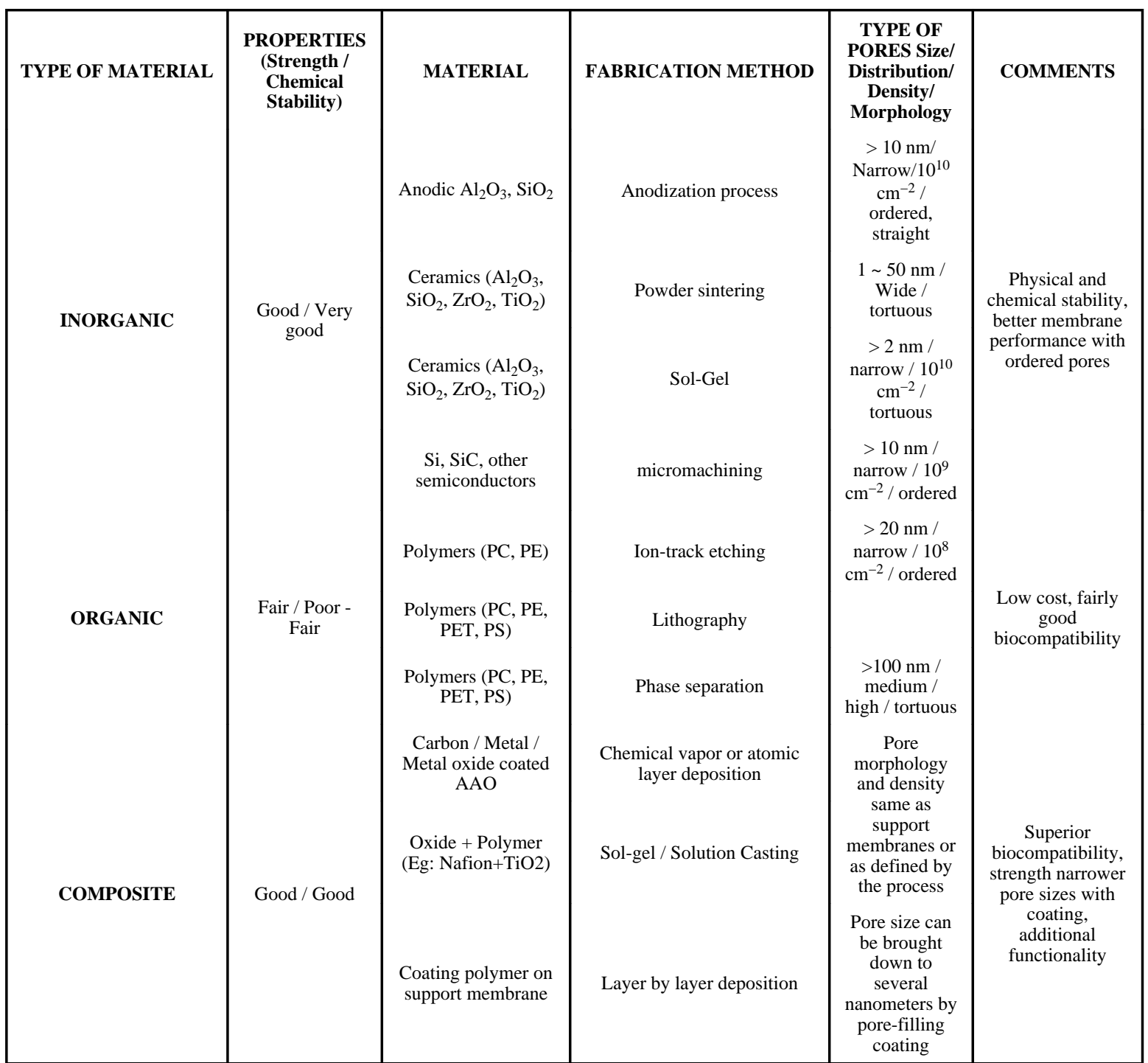

Wiley Interdiscip Rev Nanomed Nanobiotechnol. Author manuscript; available in PMC 2013 June 17. 